their own research as well as good relations with the M.R.C. at all levels. There is nothing to suggest that the Departments have had requests for help refused by the M.R.C.

But because relations between the two have been good in the past is no reason to suppose that the Rothschild proposals would have only minor effects. Throughout the world medical scientists envy British doctors for having an independent M.R.C. Its freedom to support projects it thinks worthwhile is essential to that reputation. Applicants for support for research projects know that they will be assessed objectively by practising scientists; such impartiality is not associated with Government departments. This taint-unfair though it may be-was recognized as long ago as 1918, when Lord Haldane recommended that the Medical Research Committee (as it then was) should be independent of the Department which its efforts would most nearly affect.

Furthermore, any cut in the budget under full M.R.C. control would be particularly unfortunate at present, when cuts in Government expenditure have already adversely affected university reseanch. Many university medical departments are now heavily supported by the M.R.C., and any reduction in this support might force them to close. There is not a shred of evidence that transfer of funds to the Department from the M.R.C. would have any practical advantages. The customer-contractor principle is only very marginally applicable to medical research. Medicine is not neatly divisible into basic and applied; staff need reasonable security; and research workers set a high price on their independence. Closer co-operation between the Department and the M.R.C., as suggested in the latter's memorandum to the select committee, is an obvious move in the right direction and should lead to any practicable increase in consumerorientation of medical research. It would be a tragedy to hazard a world-famous institution for doctrinaire reasons alone.

\footnotetext{
1 A Framework for Government Research and Development. London, H.M.S.O., 1971.
}

2 The Times, 27 January 1972.

\section{Brachial Plexus Birth Injury}

The roots of the brachial plexus may be damaged during difficult deliveries, particularly if extreme lateral flexion of the neck occurs or if traction on the shoulders or arms is required. This type of birth injury is not common. The frequency in one New York hospital in 1962 was 0.4 per 1,000 births. ${ }^{1}$ Complete recovery of function has been reported in $13 \%^{2}$ to $18 \%{ }^{3}$ of cases, while appreciable motor or sensory deficits remain in some $40 \%$. Minor damage to the roots of the plexus with haemorrhage into the nerve sheaths may be followed by rapid and complete recovery, whereas restoration of function may be more prolonged and less complete after avulsion of the roots from the spinal cord. Recovery within six months is nearly always total, but minor degrees of weakness or partial disturbance of function are seen in those showing recovery during the first year. ${ }^{4}$

Historically, brachial-plexus injuries have been divided into upper (Erb-Duchenne) and lower (Klumpke) plexus palsies, which produce paralysis of proximal and distal muscle groups respectively. In practice the clinical picture is seldom so well defined, particularly during the first one or two weeks of life, when the extent of the paralysis may diminish appreciably. Lesions of the upper plexus (C5-6 or $\mathrm{C} 5-6-7$ roots) are much commoner than of the whole or of the lower plexus. ${ }^{5}$ Lesions of the upper plexus have a relatively better prognosis as a recent study showed. ${ }^{4}$ Permanent weakness or deformity was reported in two out of ten infants with $\mathrm{C} 5-6$ injuries, in three out of ten with C5-6-7 root injuries, and in three out of the five in whom all the roots of the plexus had been damaged. Complete or partial recovery of function was seen in onethird of all the cases, and it occurred within the first year of life.

The fact that spontaneous recovery may occur is not an indication to delay treatment. Contractures can develop rapidly during the first six months and cause functional impairment later. Splinting does not prevent contractures unless it is combined with repeated passive movements. ${ }^{2}$ As power returns to the limb, active movements can be promoted by using techniques evolved for cerebral-palsied infants and young children. ${ }^{6}$ The later orthopaedic management of the results of injuries to the brachial plexus has been studied in detail. 7 Early galvanic stimulation of the denervated muscles has much experimental evidence to support it, ${ }^{89}$ but whether the benefits outweigh the discomfort and sometimes pain is questionable.

The physical signs associated with injury of the upper plexus are nearly always unilateral. The limb is held in shoulder adduction and internal rotation, with the elbow extended and the forearm pronated. Wrist flexion with some finger extension also occurs, to give the classical "waiter's tip" posture. This is a grossly abnormal arm position for a full-term newborn baby, whose upper limbs are generally symmetrical with flexed elbows and closed fists. The paralysed arm does not respond fully in the Moro response, 10 and the asymmetrical weakness or paralysis of the biceps muscle may be detected by the lack of normal recoil of the forearms when the elbow is passively extended and then released. The biceps jerk may be absent, but the hand grasp is usually present. Occasionally a radial nerve palsy may add to the physical signs in the forearm, wrist, and hand. An area of subcutaneous fat necrosis overlying the radial groove of the humerus offers a clue to the existence of an additional peripheral nerve injury. ${ }^{11} \mathrm{~A}$ haematoma may be palpable in the supraclavicular region, or chest movements may be reduced on the affected side, possibly with some respiratory distress, suggesting phrenic nerve injury.

Injuries to the whole plexus are more likely than partial injuries to be associated with some paralysis of the opposite arm, but the physical signs are usually asymmetrical. Damage to the spinal cord may produce signs of an upper motor neurone lesion in the lower limbs. The totally paralysed arm lacks muscle tone, tendon jerks, and sometimes sensation. It is therefore entirely limp, and its posture at all times is determined by gravity alone. Recovery of the upper roots may be rapid, and the clinical picture then becomes one of a distal palsy with wrist drop, ulnar or radial deviation of the wrist, and an absent or weak hand-grasp response. Sometimes ptosis and meiosis of the homolateral eye are seen because of damage to fibres of the sympathetic nervous system in the first thoracic root.

The basic principles of management apply to these types of palsy. When muscle imbalance produces unusual postures, it should be corrected by splinting, and all joints should be subjected to repeated passive movements. Damage to bones is likely to add to the generally poorer prognosis which these more extensive plexus injuries have, and disorders of function, both motor and sensory, and impairment of growth of the limb are likely to follow. 
1 Adler, J. B., and Patterson, R. L., Fournal of Bone and Foint Surgery, 1967, 49A, 1052.

2 Wickstrom, J., Clinical Orthopaedics, 1962, 23, 187.

2 Wickstrom, J., Clinical Orthopaedics, 1962, 23, 187.
Danos, D. E., fournal of Bone and foint Surgery, 1965, 47B, 805.

Eng, G. D., Paediatrics, 1971, 48, 18.

5 Ford, F. R., in Diseases of the Nervous System in Infancy, Childhood and Adolescence, 5th edn., p. 1063 . Springfield, Illinois, C. C. Thomas, 1966.

- Bobath, B., Developmental Medicine and Child Neurology, 1967, 9, 373.

7 Sever, J. W., Fournal of the American Medical Association, 1925, 85, 1862.

8 Osborne, S. L., Archives of Physical Medicine, 1951, 32, 523.

- Gutmann, E., and Gutmann, L., Fournal of Neurology, Neurosurgery and Psychiatry, 1944, 7, 7.

10 Forfar, J. O., British Medical Fournal, 1971, 4, 28.

11 Lightwood, R., Archives of Disease in Childhood, 1951, 26, 436.

\section{Laxative Jaundice}

In 1970 over $£ 4 \mathrm{~m}$ was spent in the United Kingdom on laxatives for self-medication, yet probably few doctors know that these preparations can cause acute and chronic hepatitis. T. B. Reynolds and colleagues ${ }^{1}$ from Los Angeles described the occurrence of an acute hepatitis-like illness in four women taking a laxative preparation, Dialose Plus, in which the active ingredient was oxyphenisatin. The jaundice improved in all cases when the laxative was discontinued and recurred in two when it was restarted. The other two patients showed prompt rises in serum transaminase levels when challenged with the drug in hospital. More cases have been reported since from America ${ }^{2} 3$ and also from Australia ${ }^{4}$ and from Britain. ${ }^{5}$ Most of the patients were middle-aged women who had been taking the drug regularly for one to two years, and they usually presented with an acute illness with jaundice, fatigue, and abdominal discomfort. The changes in liver function tests, with an increased serum transaminase, and the histological appearances of liver tissue resembled those in acute viral hepatitis.

When the drug is stopped the condition usually resolves rapidly. Otherwise hepatic damage may progress. In a more recent report Reynolds and colleagues ${ }^{6}$ describe six patients who after prolonged ingestion of laxative preparations containing oxyphenisatin developed the characteristic biochemical and histological features of the condition most commonly known as active chronic hepatitis. The histological changes in the liver were the same as those seen in hepatitis thought to be due to persisting Australia antigen in the serum or to an autoimmune reaction. Two of the patients had a positive L.E. cell test, two others had antinuclear antibodies, and three had smooth muscle antibodies. Nevertheless, they greatly improved after discontinuing oxyphenisatin and had no recrudescences, which is not in accord with the concept of autoimmunity as the perpetuating factor in this type of chronic liver disease.

These six cases of chronic active hepatitis are the first in which the disease has been attributed to a drug. There are, of course, other drug reactions in which L.E. cells are found-for example, the lupus syndrome first described after hydralazine and which may also be induced by procainamide, isoniazed, anticonvulsant drugs, and methyldopa -but hepatic abnormalities rarely occur in drug-induced lupus or in systemic lupus erythematosus. Indeed, lupoid hepatitis (the term often used to describe active chronic hepatitis when L.E. cells are present) is usually thought to be a distinct condition.

In the rat oxyphenisatin is partially absorbed from the intestine, taken up by the liver, and excreted in the bile in a form that stimulates peristalsis, but little is known about its metabolism in man. Several of the laxative preparations which were incriminated in the U.S.A. have also contained dioctyl sodium sulphosuccinate, a surface-acting agent which might enhance the absorption of oxyphenisatin and hence its toxicity. Nevertheless, jaundice has been reported after taking laxatives containing only oxyphenisatin, and the results of the challenge experiments with pure oxyphenisatin also show that it must be considered as the hepatotoxin.

Oxyphenisatin is sold over the counter in America as a component of many laxative mixtures but only a few preparations (Bydolax, Cirotyl, and Contax) in Britain contain it, and one of these has already been withdrawn from the market. The laxative ingredient of prunes is said to be oxyphenisatin, and the substance is also structurely similar to the more popular diphenylmethane laxatives, phenolphthalein and bisacodyl. But no cases of jaundice due to these compounds have yet been reported. Nevertheless, no history of a patient with hepatitis, whether acute or chronic, can be considered complete until diligent inquiry has been made into the contents of the bathroom cabinet.

\footnotetext{
1 Reynolds, T. B., Lapin, A. C., Peters, R. L., and Yamahiro, H. S., Fournal of the American Medical Association, 1970, 211, 86.

2 McHardy, G., and Balart, L. A., fournal of the American Medical Association, $1970,211,83$.

seeley, A. F., Trey, C., and Gottlieb, L. S., Gastroenterology, 1971, 60, 195.

4 Willing, R. L., and Hecker, R., Medical fournal of Australia, 1971, 1, 1179.

5 Pearson, A. J. G., Grainger, J. M., Scheuer, P. J., and McIntyre, N.,

Lancet, 1971, 1,994. Medicine, 1971, 285, 813.
}

\section{Pensions' Prospects}

In a debate on superannuation at a recent B.M.A. Council meeting one speaker said the profession was now more concerned with pensions than with pay. The brisk reaction of the Council to Sir Keith Joseph's letter in the autumn (Supplement, 27 November, p.51) rejecting the B.M.A.'s proposals towards improving N.H.S. doctors' pensions was repeated by the G.M.S. Committee (Supplement, 18 December, p.80). The National Hospital Conference in November had also made clear hospital doctors' dissatisfaction with the present situation. Altering N.H.S. doctors' superannuation is complicated by the fact that they form a relatively small part of the membership of the N.H.S. superannuation scheme. Furthermore, the future of the N.H.S. scheme will be influenced by the Government's White Paper proposals ${ }^{1}$ for reforming State pension provision.

These reforms are planned to start in April 1975. As in the existing State scheme, an individual's pension benefit will consist of two parts, namely, a basic flat rate pension and an earnings-related pension. All eligible employees will receive-as now - the basic flat rate pension but contributors who are members of a suitable occupational pension scheme can be exempt entirely from contributing to the State earnings-related scheme, in which case they will receive no benefits from that scheme. On the other hand, an employee in "non-participating employment" at present is only conrracted out of part of the graduated scheme and is still liable for graduated contributions, albeit at a reduced rate. Another important difference in principle between the proposed State earnings-related scheme and the existing graduated scheme is that while both are earnings-related the new reserve scheme will allow for pensions in payment to be increased to keep their value in a period of inflation. This does not happen now. 